\title{
Modelagem geofísica 2D a partir de dados de potencial espontâneo: uma contribuição ao estudo de zonas de fraturas hidrotermalizadas em Lavras do Sul (RS)
}

Rosales, M. J. T., UNIPAMPA, Dias, A. L. L. *, UNIPAMPA, Guimarães, M. F. S. B, UNIPAMPA, Alcalde G., UNIPAMPA, Cruz, M. F., UNIPAMPA

Copyright 2018, SBGf - Sociedade Brasileira de Geofísica

Este texto foi preparado para a apresentação no VIII Simpósio Brasileiro de Geofísica, Salinópolis, 18 a 20 de setembro de 2018. Seu conteúdo foi revisado pelo Comitê Técnico do VIII SimBGf, mas não necessariamente representa a opinião da SBGf ou de seus associados. É proibida a reprodução total ou parcial deste material para propósitos comerciais sem prévia autorização da SBGf.

\section{Abstract}

Spontaneous Potential (SP) is a natural field geophysical method widely used in mineral exploration and groundwater study, based on the measurement of a potential difference between two electrodes introduced into the soil.

This research aims to demonstrate the efficiency of the Spontaneous Potential Method (SP) in detailed scale geophysical surveys in Lavras do Sul Intrusive Complex (CILS), in an area of $4.8 \mathrm{~km}^{2}$. It was used resistivity data from a Vertical Electrical Survey (VES), located near the SP profiles, in order to correlate the geological and geometric parameters with the results obtained in the SP models. The results of the qualitative interpretation of the Spontaneous Potential data obtained for the E-E 'and D$D^{\prime}$ profiles indicates the presence of anomalies of the order of $+15 \mathrm{mV}$ to $-5 \mathrm{mV}$, following in general, the flow direction of water coming from the streams suggesting the presence of fractures filled with water. The 2D modeling of the Spontaneous Potential data corresponds to the E$\mathrm{E}^{\prime}$ profile oriented according to WWN-EES, D-D' profile oriented according to NW-SE and F-F' profile oriented according to SW-NE. According to the proposed geophysical models, anomalies with a negative signal of low amplitude around $-5 \mathrm{mV}$ may be associated with the presence and/or accumulation of water in zones of fractures, almost outcropping, that can extend from the first meters in surface, presenting continuity in depth.

\section{Introdução}

A área da pesquisa se encontra inserida na porção central do Complexo Intrusivo Lavras do Sul (CILS), compreendendo rochas pertencentes ao enclave central, caracterizadas por Biotita (Bt) - Granodiorito de aproximadamente 601 - $599 \mathrm{Ma}$, e de Anfibólio (Amp) Biotita (Bt) (Gastal et al., 2006). A área possui 4,8 km² e se situa na localidade rural do Cerrito, em torno de $2 \mathrm{~km}$ a Leste da cidade de Lavras do Sul (RS), delimitada geograficamente pelas longitudes UTM: $223680 \mathrm{~W}-$ 223920 W, e as latitudes UTM: 6589110 S - 6588910 S.

A finalidade da pesquisa de maneira geral é demonstrar o grau de eficiência da aplicação do Método de Potencial Espontâneo (SP) em levantamentos geofísicos em escala de detalhe (1:2000), com um intervalo de amostragem de aproximadamente 15 metros, tendo como objetivo principal esclarecer questões relacionadas com a caracterização geológica e geofísica, visando à identificação e caracterização de zonas com alteração hidrotermal associadas com zonas de falhas e/ou de fraturas, com perspectivas de hospedarem presença de mineralização metálica em sulfetos disseminados, e por consequência mineralização aurífera. Para a aplicação do método de SP, foi escolhido um setor da área de estudo, caracterizado pela presença de um solo residual provindo de rochas granodioríticas, onde se observam fragmentos de rochas alteradas oriundas de rejeitos de antigas trincheiras. Os resultados da interpretação qualitativa dos dados de SP, indicam a presença de intervalos de valores de SP de $+15 \mathrm{mV}$ a $-5 \mathrm{mV}$, nos perfis E-E', D-D' e F-F', correlacionando-se de maneira geral, com uma direção de fluxo de água proveniente dos riachos (zona de drenagem) vindo de áreas de cotas mais elevadas para as áreas mais baixas, sugerindo a presença de fraturas preenchidas com água, uma vez que trata-se de anomalias de pequena amplitude, se comparadas com as que poderiam esperar-se para a presença de mineralização metálica em veios de quartzitos. Foi utlizado dados de resistividade oriundos de uma Sondagem Elétrica Vertical (SEV), localizada próximo aos perfis de SP, a fim de correlacionar os parâmetros geolétricos e geométricos com os resultados obtidos nos modelos de SP.

Resultados obtidos a partir do mapa da anomalia de SP, e da interpretação quantitativa dos modelos geofísicos geológicos obtidos para os perfis E-E' e D-D', sugere a presença de água nas zonas de fraturas das rochas granodioríticas.

\section{Metodologia}

O Potencial Espontâneo (SP) é um método geofísico de campo natural muito utilizado em prospecção mineral e no estudo de águas subterrâneas, baseando-se na medição de uma diferença de potencial entre dois eletrodos introduzidos no terreno. Segundo Orellana, 1972, este método baseia-se no fato de que, sob certas condições geológicas, na presença de heterogeneidades condutoras do subsolo, estas são polarizadas, tornandose verdadeiras baterias elétricas, que originam correntes elétricas no subsolo. Essas correntes produzem uma distribuição de potenciais observáveis na superfície do terreno, o que pode revelar a presença de um corpo polarizado. Entre os corpos que podem apresentar este fenômeno estão os depósitos de alguns sulfetos (pirita, pirrotita, calcocita, etc.), certos óxidos (magnetita, pirolusite), minerais ou rochas carbonatadas (grafite, antracito) e a presença de fluxo de água. A anomalia de SP produzida pela presença de fluxo de água 
subterrânea tende a ter um comportamento mais suavizado e com uma menor amplitude do sinal, se comparada com as anomalias produzidas por corpos constituídos por sulfetos e óxidos metálicos.

O SP é um método passivo, ou seja, não necessita da injeção de corrente elétrica para que o campo elétrico seja gerado. O potencial natural ou espontâneo medido em milivolts $(\mathrm{mV})$ é causado pela atividade eletroquímica ou eletromecânica produzida na presença de corpos metálicos com diferentes propriedades elétricas, principalmente com variações de resistividade, produzindo de maneira geral anomalias negativas. Uma outra resposta de SP é oriunda do denominado "potencial de fluxo", o qual é devido ao movimento de fluidos através dos poros e descontinuidades das rochas, sendo também denominado de potencial de eletrofiltração. A passagem de um eletrólito através de uma membrana porosa produz uma diferença de potencial entre os dois lados da mesma. Considerando a porosidade do substrato como uma rede de capilares por onde percolam as águas de subsuperfície, então o comportamento do substrato pode ser visto como uma membrana. A medida deste tipo de potencial tem sido utilizada para detecção de caminhos preferenciais da água subterrânea, auxiliando no mapeamento de divisores d'água e na direção do fluxo. Em áreas com relevo acidentado onde os aquíferos mantêm uma dinâmica controlada pela gravidade em função dos taludes da topografia, existe uma resposta de potencial elétrico natural originando uma anomalia negativa coincidindo espacialmente com a área de descarga do aquífero em zonas caracterizadas por altos topográficos. A reposta originando uma anomalia positiva no contexto anterior é válida para as áreas de recarga do aquífero em zonas onde predominam a presença de baixos topográficos.

O levantamento de Potencial Natural Elétrico foi realizado na área de estudo com um espaçamento regular de aproximadamente 15 metros de abertura do dipolo de medição, contando com um total de 91 pontos físicos medidos, utilizando a técnica de gradiente. Esta técnica consiste em medir sucessivamente as diferenças de potencial entre dois pontos contíguos de um perfil em polígonos fechados, tendo a grande vantagem de fornecer um controle da medição, levando em consideração o erro médio quadrático dos dados do levantamento. Após realizada a medição entre dois pontos contíguos, o arranjo é deslocado ao longo da linha, normalmente a intervalos regulares.

O algoritmo utilizado foi projetado para modelagem direta e inversa de campos de potencial espontâneo natural (SP) que permite simular os padrões geoelétricos simultaneamente para até 29 condutores prismáticos polarizados eletrostaticamente (Dmitriev, 2012). O software ZONDSP2Dp (Zond Software, 2018) destina-se à interpretação bidimensional dos dados adquiridos pelo método do campo elétrico natural. $\mathrm{O}$ ambiente do modelo é representado por um conjunto de corpos que simula uma seção poligonal. $O$ algoritmo matemático é baseado no ajuste dos dados pelo método dos mínimos quadrados e permite simular em modo automático padrões de resistividades que podem conter condutores polarizados eletrostaticamente, apresentando diferentes geometrias. Os dados de resistividade aparente obtidos em campo foram a partir de uma Sondagem Elétrica Vertical (SEV) com o arranjo Schlumberger, posicionada na porção central da área de estudo, próxima ao perfil E-E'. Os parâmetros geoelétricos e geométricos obtidos a partir da inversão da curva de resistividade aparente serviram de subsídio para correlacionar os padrões de resistividade dos condutores polarizados nos modelos de SP obtidos para os perfis E-E', D-D' e F-F'. Para a inversão (1D) dos dados de resistividade aparente oriunda da SEV, foi utilizado o programa IPI2Win 3.0.1a. elaborado pela Universidade de Moscou, 2003.

\section{Área de Estudo}

A Figura 5 ilustra o mapa geológico simplificado da área de estudo compreendendo as rochas pertencentes ao enclave central, caracterizadas por Biotita (Bt) Granodiorito de aproximadamente 601 - $599 \mathrm{Ma}$, e de Anfibólio (Amp) - Biotita (Bt) - Monzogranito, de idade aproximada de $604 \mathrm{Ma}$. Na área é destacável a intensa ação da tectônica rúptil apresentando estruturas radiais associadas à presença de falhas e fraturas, que servem de principais condutos de acesso das mineralizações. Observa-se o posicionamento dos perfis de SP, E-E', DD' e F-F' que foram objeto da modelagem.

A Figura 6 se corresponde com uma imagem que mostra um afloramento de rocha granodiorítica pouco alterada na porção Leste da área de estudo. É composta por Kfeldspato, plagioclásio, quartzo, biotita e anfibólio. Os feldspatóides são euédricos e possuem granulação média. O quartzo e a biotita apresentam-se na fração fina à média e os grãos são subédricos. Se destaca a presença de duas direções preferenciais de fraturas com uma disposição ortogonal, isto é, na direção $\mathrm{N} 75^{\circ} \mathrm{W}$ e na direção $\mathrm{N} 30^{\circ} \mathrm{E}$. Observa-se a presença de um fluxo de água superficial acompanhando a direção $\mathrm{N} 30^{\circ} \mathrm{E}$.

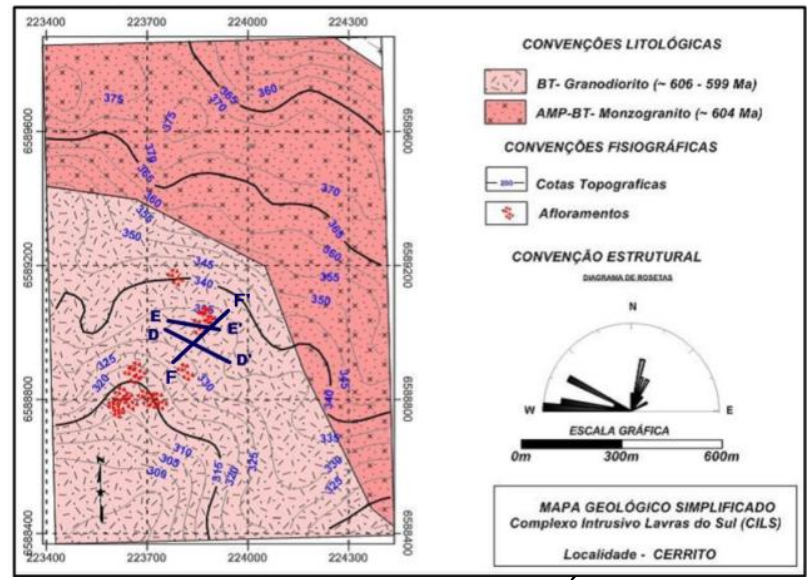

Figura 5 - Mapa geológico da Área de Estudo (modificado de Gastal et al., 2006) 


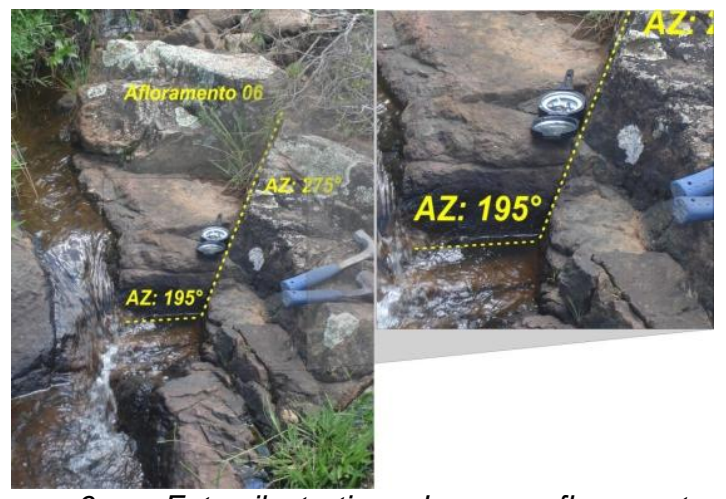

Figura 6 - Foto ilustrativa de um afloramento de Granodiorito porfirítico, com a linha amarela indicando a direção das fraturas.

\section{Integração de dados de SP e de resistividade na área de estudo}

No mapa integrado de anomalias de Potencial Espontâneo (SP) na área de estudo (Figura 7), observase a malha de pontos medidos de dados SP, a SEV 02 e os perfis E-E', D-D' e F-F' que foram objeto de modelagem. É importante ressaltar a presença de zonas de drenagem dispostas na direção NE e que cortam os perfis E-E' e D-D', e a presença de afloramentos de Granodioritos na porção Leste. A área se encontra caracterizada por um relevo abrupto com as cotas mais altas em torno de 335 metros de altitude na porção Norte.

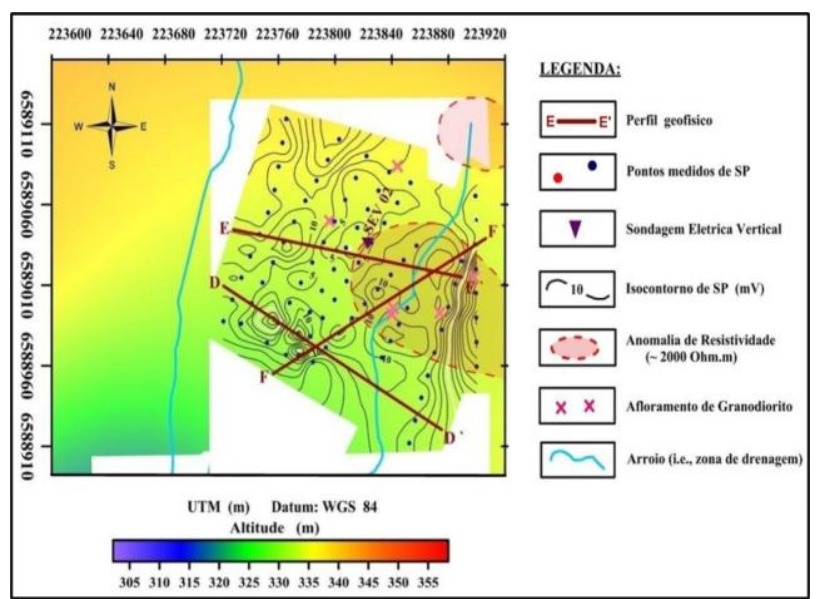

Figura 7 - Mapa esquemático integrado da Anomalia de Potencial Espontâneo (SP), mostrando a malha de pontos medidos, os Perfis E-E', D-D', F-F', o posicionamento da SEV 02 e a localização de afloramentos.

E notório ressaltar na porção Norte do mapa, caracterizada por altos topográficos, a presença de dois trends anômalos com valores na ordem de $+14 \mathrm{mV}$, com uma orientação segundo NE-SW apresentando uma disposição espacial quase paralela, e acompanhando de certa maneira a direção de fluxo de água proveniente dos riachos ou arroios, vindo das zonas de cotas mais elevadas para as zonas de cotas mais baixas.
No extremo Leste destaca-se a presença de uma anomalia com formato dipolar, com valores de SP variando de $-5 \mathrm{mV} \mathrm{a}+15 \mathrm{mV}$, associadas à presença do acúmulo e/ou movimentação de água nas fraturas das rochas aflorantes (i.e., Bt Granodiorito ( 601 - $599 \mathrm{Ma}$ )).

A Figura 8 mostra os gráficos integrados da Anomalia de Potencial Espontâneo (SP) conjuntamente com o relevo topográfico oriundo do ASTER-GDEM ao longo dos Perfis E-E', D-D' e F-F' presentes na área de estudo.

No perfil E-E' (WWN-EES) observa-se um comportamento mais suave da anomalia de SP, variando num intervalo de $+15 \mathrm{mV}$ a $+1 \mathrm{mV}$. O perfil se encontra posicionado em um mesmo patamar topográfico em torno de 333 metros de altitude. Na porção Leste do perfil destaca-se a presença de afloramentos de Granodiorito.

No perfil D-D' (NW-SE), destaca-se valores de SP com amplitude em torno de $+15 \mathrm{mV}$, na presença de um dipolo com um sinal negativo vizinho da ordem de $-5 \mathrm{mV}$. Este comportamento poderia estar associado a presença de uma zona de fraturas com presença de água.

No perfil F-F' (SW-NE), a anomalia de SP varia suavemente no intervalo de $+14 \mathrm{mV}$ a $+3 \mathrm{mV}$. Se caracteriza pela presença de uma variação abrupta do relevo topográfico na direção NE, na ordem de 329 a 334 metros.

Segundo Rosales et al., (2016), anomalias negativas de pequena amplitude de SP se encontram associadas à presença de zonas de fraturas com água e corroboram a ideia de que o Potássio e o Urânio podem ter sido lixiviados mediante a circulação das águas de zonas mais altas do relevo topográfico para as zonas mais baixas.

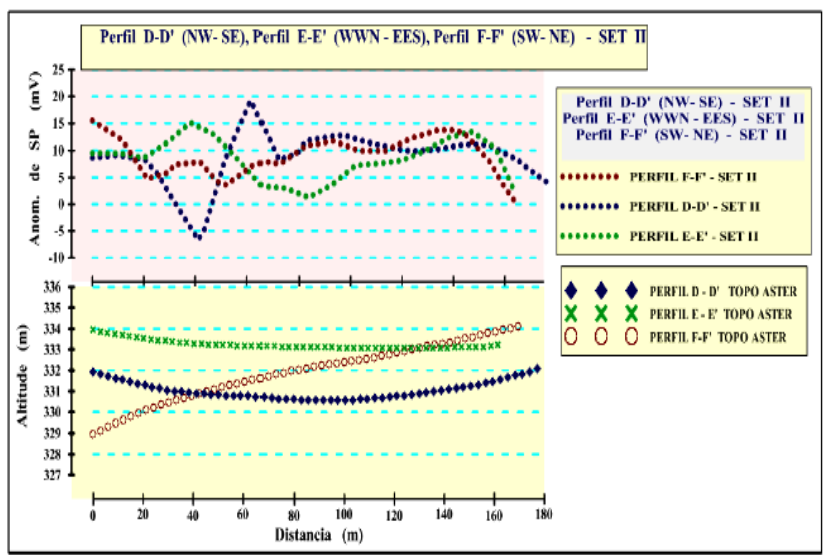

Figura 8 - Gráficos integrados da Anomalia de Potencial Espontâneo (SP) dos perfis $E-E^{\prime}, \quad D-D^{\prime}$ e $F-F^{\prime}$ conjuntamente com o relevo topográfico.

A Figuras 9 e 10 mostram as curvas de campo de resistividade aparente oriundas dos dados adquiridos da SEV 02 e a inversão dos dados de SEV, conforme 4 camadas geoelétricas $(\rho 1>\rho 2<\rho 3<\rho 4)$. A interpretação do modelo obtido é representada na Tabela 1. 


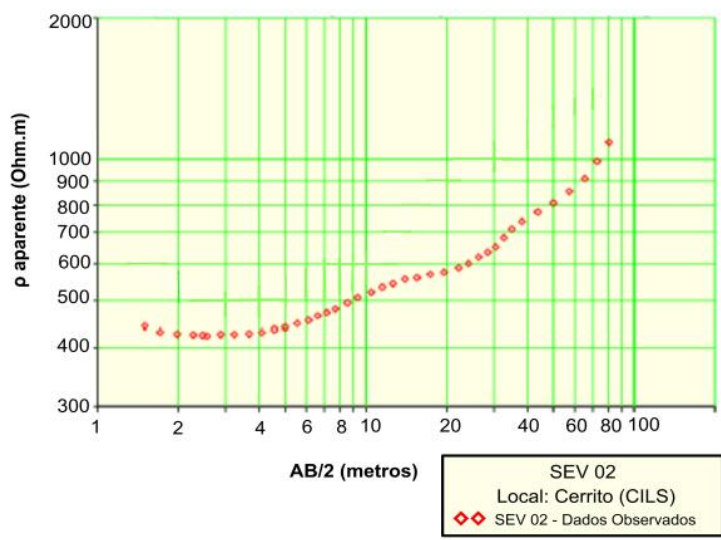

Figura 9 - Curva de resistividade aparente da SEV 02.

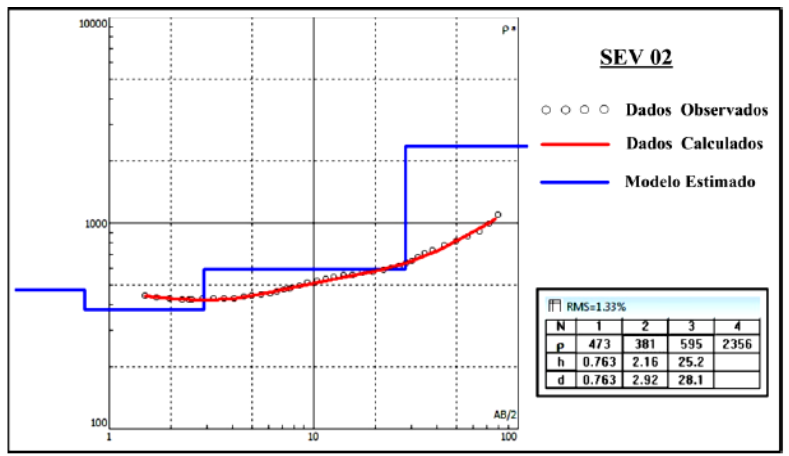

Figura 10 - Modelo de inversão para a SEV 02 conforme quatro camadas geoelétricas.

Tabela 1 - Dados de inversão da SEV e sua interpretação.

\begin{tabular}{|c|c|c|c|}
\hline Camada & Espessura (m) & Resistividade (Ohm.m) & Interpretação \\
\hline 1 & $0-0.763$ & 473 & Solo Seco \\
\hline 2 & $0.763-2.16$ & 381 & Solo Úmido \\
\hline 3 & $2.16-25.2$ & 595 & $\begin{array}{c}\text { Granodiorito Alterado } \\
(\sim 606-599 \mathrm{Ma})\end{array}$ \\
\hline 4 & & 2356 & $\begin{array}{c}\text { Granodiorito } \\
(\sim 606-599 \mathrm{Ma})\end{array}$ \\
\hline
\end{tabular}

A coluna geoelétrica (Figura 11) confeccionada a partir da interpretação do modelo, destaca a presença de 3 camadas geoelétricas com diferentes espessuras e com valores de resistividade variando em torno de 300 a 600 Ohm.m, sendo que as duas primeiras camadas até aproximadamente 3 metros de profundidade, caracteriza a presença de um solo residual com diferentes graus de umidade. A partir dos 3 metros de profundidade destacase a presença de uma espessa camada ( 23 metros) com resistividade de $595 \mathrm{Ohm} . \mathrm{m}$ que caracteriza o topo do Granodiorito com diferentes graus de alteração.

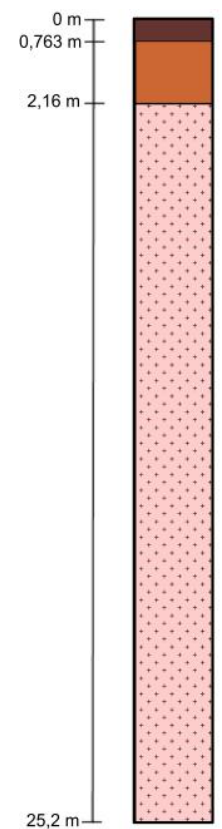

\section{LEGENDA}

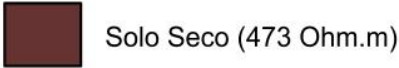

Solo Úmido (381 Ohm.m)

Granodiorito Alterado (595 Ohm.m)

Figura 11 - Coluna geoelétrica obtida a partir da inversão dos dados de SEV.

\section{Interpretação dos modelos 2D dos dados de SP}

As Figuras 12, 13 e 14 ilustram os modelos geofísicos 2D obtidos a partir da interpretação dos dados de SP para os perfis E-E', D-D' e F-F' orientados segundo WWN-EES, NW-SE e SW-NE respectivamente, localizados na área de estudo. 


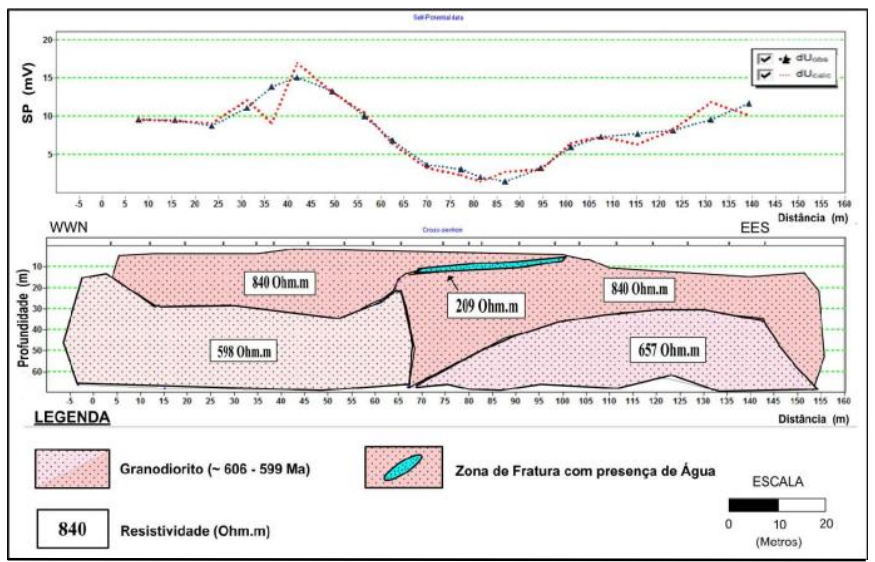

Figura 9 - Modelo geofísico geológico proposto para Perfil E-E' orientado segundo WWN-EES, localizado na área de estudo.

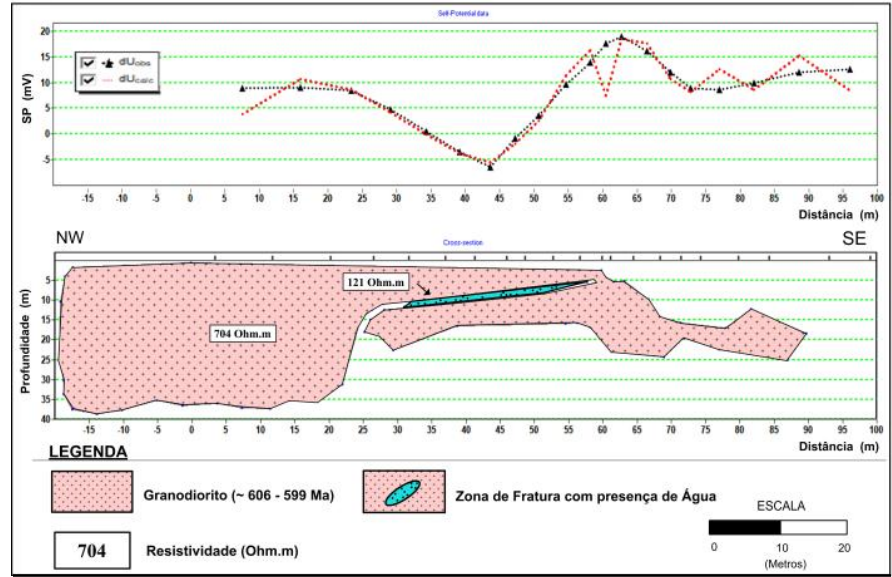

Figura 10 - Modelo geofísico geológico proposto para Perfil D-D' orientado segundo NW-SE, localizado na área de estudo.

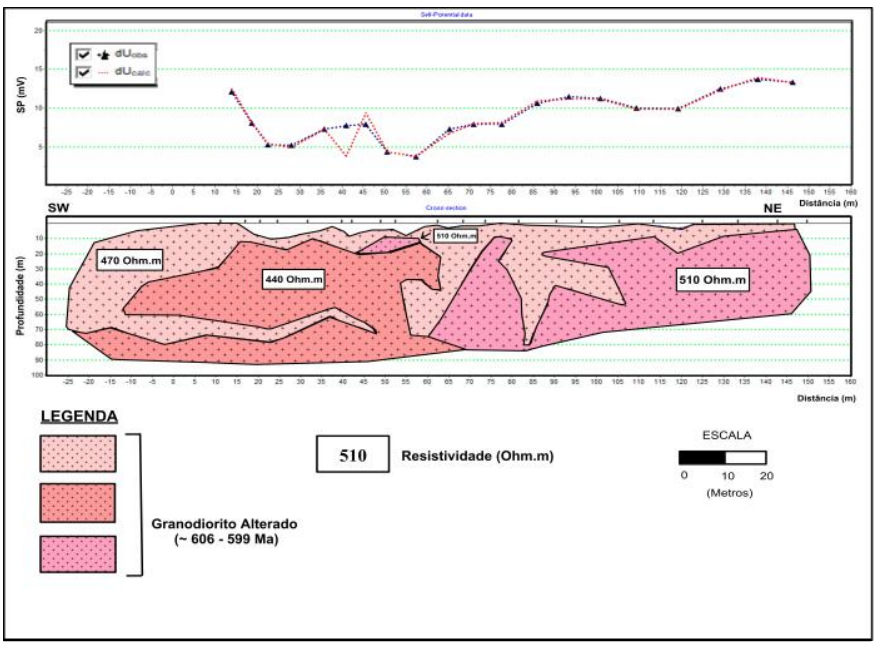

Figura 11 - Modelo geofísico geológico proposto para Perfil F-F' orientado segundo SW-NE, localizado na área de estudo.

O modelo geofísico obtido para o perfil E-E' (WWN EES) destaca a presença de um embasamento constituído de rochas granodioríticas com diferentes valores de resistividade que variam de 840 Ohm.m a 590 Ohm.m, o que sugere que nesta direção do perfil a rocha tenha sido afetada por zonas de fraturas e/ou falhas. $\mathrm{Na}$ porção Leste do modelo destaca-se a presença de uma zona de fratura com um mergulho sub-horizontal caracterizada por valores de resistividade de 209 Ohm.m, 
sugerindo a presença e movimentação de águas subterrâneas.

O modelo geofísico obtido para o perfil D-D' (NW - SE) destaca a presença de um embasamento alterado constituído de rocha granodiorítica com valor de resistividade de aproximadamente 704 Ohm.m. Na porção central do perfil destaca-se a presença de uma zona de fraturas caracterizada por uma resistividade de 121 Ohm.m, sugerindo a presença e movimentação de águas subterrâneas. É notório destacar que na direção deste perfil não existem ocorrências de afloramentos e ele se encontra posicionado em um zona topográfica inferior ao do perfil E-E', o que poderia explicar a presença de um patamar de resistividades inferior ao mesmo. É importante ressaltar tanto para o modelo do perfil E-E' como para o modelo do perfil D-D', os quais apresentam uma disposição em campo na direção Leste, que ambos os perfis cortam uma zona de drenagem na direção N-S, o que sugere que a movimentação de água superficial ao longo das fraturas observadas nas rochas aflorantes possa ter continuidade em profundidade.

No modelo geofísico obtido para o perfil F-F' (SW-NE) é destacado a presença de um embasamento constituído de rochas granodioríticas com diferentes valores de resistividade que variam em torno de 510 até 440 Ohm.m. É notório destacar que neste modelo o topo do embasamento granodiorítico apresenta fortes irregularidades no formato geométrico, o que sugere diferentes graus de alteração. O patamar inferior de resistividade ( 510 Ohm.m - 440 Ohm.m), em relação aos outros perfis, pode ser explicado pela presença de uma variação abrupta do relevo topográfico da direção NE na ordem de 329 a 334 metros. Segundo o trabalho de Medeiros et. al., 1999, no estudo de Potencial Elétrico Espontâneo, ao longo de seções transversais, em aquíferos fissurais em rochas cristalinas do Estado da Bahia, os perfis de SP apresentam ordens de grandeza relacionadas à amplitude das anomalias em torno de -15 a $-50 \mathrm{mV}$ e mostram, portanto, um padrão semelhante aos apresentados na presente pesquisa, sugerindo que as anomalias de SP dos perfis E-E' e D-D' poderiam ser originadas pelo fluxo de água subterrânea.

\section{Discussões e Conclusão}

A modelagem de dados de SP oferece uma ferramenta muito eficiente para o esclarecimento e/ou entendimento de questões relacionadas com a geometria e os processos vinculados com zonas de fraturas, onde exista acúmulo e movimentação de água subterrânea, e comprovadamente possam existir indícios de mineralização metálica associadas a processos hidrotermais.

Conforme os modelos obtidos para os perfis E-E' e D-D' conclui-se que o embasamento constituído por rochas granodioríticas na área de estudo se encontra afetado por diferentes graus de alteração e caracterizado pela presença de zonas de fraturas observadas em afloramentos, as quais apresentam continuidade em profundidade até aproximadamente 15 metros.
Os resultados obtidos se mostram consistentes e alentadores do ponto de vista de interpretação geológico - geofísico, integrando os dados dos levantamentos em escala de detalhe, provindo de medições de Potencial Espontâneo e Sondagem Elétrica Vertical, visando à identificação e a caracterização de zonas com alteração hidrotermal associadas com zonas de falhas e/ou de fraturas, com perspectivas de hospedarem presença de mineralização metálica em sulfetos disseminados, e por consequência mineralização aurífera. Conforme os modelos geofísicos propostos para os perfis E-E' e D-D' obtidos a partir da inversão 2D dos dados de Potencial Espontâneo (SP), pode-se concluir que anomalias com sinal negativo de baixa amplitude em torno de $-5 \mathrm{mV}$, poderiam estar associadas a presença e/ou acúmulo de água em zonas de fraturas, quase aflorantes, que podem estender-se desde os primeiros metros em superfície, apresentando uma continuidade em profundidade.

\section{Referências}

Alex Kaminsky, 2001-2018, Zond Software.

Aster Global DEM Validation Summary Report. Disponível em: http://www.gdem.aster.ersdac.or.jp/index.JTPmede

Bobachev, A., Modin, I., Shevnin, V., 2003. IPI2Win software. Versión 3.0.1a. Geoscan-M Ltd. Moscow State University. Russia.

Dmitriev, A. N., 2012. Forward and inverse self-potential modeling: a new approach. Russian Geology and Geophysics. 53: 611-622.

Gastal, M.C.P., Lafon, J.M., Ferreira, F.J.F., Magro, F.U.S., Remus, M.V.D. \& Sommer, C.A., 2006. Reinterpretação do Complexo Intrusivo Lavras do Sul RS, de acordo com os sistemas vulcano-plutônicos de subsidência. Parte I: Geologia, geofísica e geocronologia (207Pb/206Pb e206Pb/238U). Revista Brasileira de Geociências, v. 36: 109-124.

Medeiros, W.E., Lima, O. A. L. 1999. Origem do Potencial Elétrico Espontâneo em rochas cristalinas fraturadas e sua utilização na locação de poços. Brazilian Journal of Geophysics, Vol. 17(2,3).

Orellana, E. 1972. Prospeccion geoeletrica en corriente continua. Madrid: Paraninfo, $523 \mathrm{p}$.

Rosales, M. J. T., Lopes, H. S., Leandro, C. G., 2016. Inversão de dados de Potencial Espontâneo (SP) e sua aplicação ao estudo de zonas hidrotermais associadas à mineralização aurífera, no Complexo Granítico de Lavras do Sul (RS) - Brasil.. In: VIII Congreso Uruguayo de Geología, I Simposio de Recursos Hídricos de la Cuenca del Plata., 2016, Montevideo, Uruguay.. In: VIII Congreso Uruguayo de Geología, I Simposio de Recursos Hídricos de la Cuenca del Plata... Montevideo, Uruguay.: EASY PLANNERS, 2016. Montevideo, Uruguay.: EASY PLANNERS, 2016. 